\title{
RESEARCH
}

Open Access

\section{Ovarian carcinoma in patients with BRCA mutation - a correlation between the growing pattern of peritoneal implants evaluated by CT/MRI and the genotype BRCA1 and BRCA2}

Ana Catarina Vieira ${ }^{1,2^{*}}$, Natalie Antunes ${ }^{3}$, Eduarda Damasceno ${ }^{4}$, Madalena Ramalho ${ }^{5}$, Susana Esteves ${ }^{6}$, Fátima Vaz ${ }^{7}$ Ana Félix ${ }^{8 \dagger}$ and Teresa Margarida Cunha ${ }^{1+}$

\begin{abstract}
Background: Ovarian cancer is the leading cause of death from gynecologic cancer. The risk of developing ovarian cancer is significantly increased in patients that carry a genetic mutation of tumor suppressor gene BRCA1 or BRCA2. The majority of BRCA-associated ovarian/fallopian tube cancers are high-grade serous carcinomas (HGSC). The recognition of patterns of disease is crucial to identify distinctive imaging features that could be useful for predicting prognosis and therapeutic response.

Results: An institutional review board-approved retrospective study was performed and included 34patients (23 BRCA-mutated and 11 BRCA wild-type) with HGSC FIGO III/IV who underwent pre-operative or pre-chemotherapy contrast-enhanced CT/MRI of the abdomen and pelvis between January 2003 and December 2017. Three radiologists independently reviewed the imaging studies and looked for qualitative features of the primary tumor and peritoneal metastases (nodular versus infiltrative pattern). Two pathologists also assessed the histopathologic characteristics of the surgical specimens, with emphasis on the growth pattern of metastatic deposits (expansive/ nodular and infiltrative) and inflammatory infiltrate (intra- and/or peritumoral).

No significant associations were found between the different groups of patients (BRCA1-mutant HGSC, BRCA2mutant HGSC. and BRCA wild-type) and CT/MRI features of ovarian tumors, morphology of peritoneal metastasis, and pathologic characteristics.
\end{abstract}

Conclusion: Identification of specific imaging and pathologic features is important to pursue an optimal personalized cancer treatment strategy and to develop precision medicine in the future.

Keywords: Cancer, Ovary, High-grade serous carcinoma, Peritoneal metastases, BRCA1, BRCA2

* Correspondence: anacfsvieira@gmail.com

${ }^{\dagger}$ Ana Félix and Teresa Margarida Cunha contributed equally to this work.

${ }^{1}$ Radiology Department, Instituto Português de Oncologia de Lisboa

Francisco Gentil, Lisbon, Portugal

${ }^{2}$ Radiology Department, Hospital do Espírito Santo de Évora, EPE, Évora,

Portugal

Full list of author information is available at the end of the article

(0) The Author(s). 2020 Open Access This article is licensed under a Creative Commons Attribution 4.0 International License, which permits use, sharing, adaptation, distribution and reproduction in any medium or format, as long as you give appropriate credit to the original author(s) and the source, provide a link to the Creative Commons licence, and indicate if changes were made. The images or other third party material in this article are included in the article's Creative Commons licence, unless indicated otherwise in a credit line to the material. If material is not included in the article's Creative Commons licence and your intended use is not permitted by statutory regulation or exceeds the permitted use, you will need to obtain permission directly from the copyright holder. To view a copy of this licence, visit http://creativecommons.org/licenses/by/4.0/. 


\section{Background}

Ovarian cancer remains the leading cause of death from gynecologic cancer [1, 2]. Epithelial carcinoma of the ovary represents a public health problem because of its considerable impact on morbidity and mortality being the 7th most common cancer and the 8th cause of cancer-related death in women worldwide [2-4].

Although cancer-related deaths have been decreasing in the last 5 decades for the majority of solid cancers, ovarian cancer survival does not show a significant improvement since about 1980 [3].

There is a multiplicity of histologic types of ovarian cancer; however, $90 \%$ of these are epithelial carcinomas [5].

The lifetime risk of ovarian epithelial carcinoma is $0.7 \%$ in sporadic cases; however, this risk is significantly increased in patients with familial or genetic predisposition $(10-40 \%)[4,6]$.

There are multiple well-established risk factors for ovarian cancer. One of the most important is BRCA germline mutation; nevertheless, other genomic mutations have been linked to this type of cancer as BARD1, CHEK2, MRE11A, RAD50, PALB2, and ATM mutations. Other known risk factors include familial history of cancer, early menarche, late menopause, nulliparous, obesity, diabetes, and smoking [3].

A scrupulous histopathologic evaluation of BRCA positive women has revealed that the majority of ovarian cancers in these patients are high-grade serous carcinomas (HGSC) [7]. In fact, they are associated with BRCA mutations in $15-17 \%$ of cases [4].

Thus, patients that carry the genetic mutation of the tumor suppressor gene BRCA1 or BRCA2 have a greater risk of developing a wide range of cancers, in particular ovarian and breast cancer. Moreover, 90\% of BRCAassociated ovarian cancers will be HGSC of higher grade than sporadic ones $[4,8]$.

Approximately $40 \%$ of BRCA1 mutated patients may develop ovarian cancer and 17\% of BRCA2-mutated patients may develop ovarian cancer by the age of 70 years old $[4,6,7]$.

This increased risk is well established and has a paramount importance on cancer screening in this particular group of patients.

HGSC is also almost invariably associated with P53 mutation and genomic instability due to deficient DNA repair [3].

The recognition of patterns of disease is crucial to identify distinctive imaging features between disease forms that could be useful for predicting prognosis and therapeutic response and enabling us to provide valuable information for a possible personalized therapy and family counseling in the future $[1,9]$.

BRCA1 and BRCA2 are tumor suppressor genes located on chromosomes 17 and 13, respectively. These genes encode proteins that are responsible for genomic stability. The BRCA-mutated patients are incapable of repairing DNA damage that will lead to uncontrolled cell proliferation with tumor development [4].

Recent studies, both from the same group, suggested that the pattern of peritoneal disease distribution in HGSC is influenced by the BRCA mutation and that these variables may have an impact on treatment response and prognosis [10].

Computed tomography $(\mathrm{CT})$ and magnetic resonance imaging (MRI) play a decisive role in the diagnosis and staging of ovarian neoplasms. Contrast-enhanced CT as an accuracy of $94 \%$ for ovarian cancer staging and is the current imaging modality of choice in the preoperative assessment of HGSG. It is an accurate method for identifying sites of disease involvement and for the assessment of resectability of ovarian cancer MRI has a reported equivalent accuracy to $\mathrm{CT}$ in predicting peritoneal tumor spread $[11,12]$.

Previous studies indicate that the mutation of the BRCA gene produces characteristic differences in the histology of ovarian serous carcinoma, but it is not clear whether these translate into morphological and radiological manifestations and, if so, that these can be safely identified. Furthermore, although some studies have demonstrated that CT features may help as useful predictors of cytoreductive outcome in HGSC, it is not known if these features differ depending on $B R C A$ mutation status nor between different groups of BRCAmutated patients (BRCA 1/2, ) [13-16].

Given the potential prognostic and therapeutic implications of BRCA mutation status is important to pursue imaging or histopathologic differences between distinct genetic mutated and wild-type ovarian cancers that could help us to provide an optimal personalized cancer treatment strategy. Our goal is to review the radiological and pathological features of all BRCA-associated HGSC cases while comparing them to a control group of nonBRCA-associated HGSC, aiming to investigate a possible association of its morphological patterns with the BRCA mutation status/type of mutation.

\section{Methods}

The Institutional Review Board approval was obtained, and written informed consent was waived by the Institutional Review Board (UIC/1199).

A retrospective study was performed with patients followed at our institution between January 1, 2003, and December 31, 2017, with preoperative or prechemotherapy contrast-enhanced CT/MRI of abdomen and pelvis with the following inclusion criteria: all histopathologically confirmed HGSC; diagnosed at FIGO stage III or IV; with a BRCA positive diagnostic genetic test. The control group was selected from patients with 
diagnostic genetic test negative for BRCA, with pathologically confirmed HGSC; FIGO stage III or IV.

Demographic, clinical, and pathologic data were collected for all patients (Table 1).

All patients underwent surgical resection according to our institutional surgical standardized procedure that include total abdominal hysterectomy, bilateral salpingo-oophorectomy, omentectomy, and lymphadenectomy. An experienced oncologic pathologist from our institution reviewed all final surgical specimens and used the World Health Organization criteria to diagnose HGSC [17].

From 2003 to 2017, patients were referred for genetic counseling and BRCA testing based on at least one of the following indications: a family member with a known $B R C A$ mutation; personal or family history of cancers with characteristics that suggest they are related to $B R C A$ mutation (breast cancers in young patients, triplenegative breast cancers in women younger than 60 years, bilateral breast cancers, multiple cases of breast cancer in a family, ovarian cancer, male breast cancer); individuals of Ashkenazi Jewish ancestry [1].

CT scans were performed in a multi-detector CT scanner with 64 detector rows and MRI examinations were obtained in a $1.5 \mathrm{~T}$ device. CT images were acquired during a breath-hold with the following acquisition parameters: $120 \mathrm{kVp}$; automatic milliampere setting, from 240 to $400 \mathrm{~mA}$; section thickness of $5 \mathrm{~mm}$. All patients received $120 \mathrm{~mL}$ of intravenous contrast material with the time delay from contrast agent injection to image acquisition of $70 \mathrm{~s}$. MRI images were acquired on a Phillips $1.5 \mathrm{~T}$ equipment in different planes: axial $\mathrm{T} 2$ of the $\mathrm{ab}-$ domen and DWI of the abdomen; axial T1 and T2 of the ovary, axial and sagittal T2 of the pelvis, DWI of the pelvis, and $\mathrm{T} 1$ fat sat dynamic after contrast medium.

The CT scans and MRI's were independently and retrospectively interpreted by 3 radiologists with good inter-observer agreement. Each reader recorded the presence of a primary ovarian/fallopian tube tumor as well as peritoneal metastasis and documented the morphological characteristics of the peritoneal metastasis as nodular (round appearance) (Fig. 1) or infiltrative (poorly defined borders) (Fig. 2).

Histopathologic characteristics were also reviewed on the surgical specimens and the presence of heavy intraepithelial lymphocytes infiltration versus absence of or sparse infiltration in the tumor, and its metastasis was evaluated and classified as present or absent. The predominant pattern of the metastatic growth in omentum (expansile versus infiltrating) was also assessed (Fig. 3a, b) accordingly with the criteria proposed by Hussein el al [18].

Associations between clinical, pathologic and imaging characteristics versus $B R C A$ mutation status were evaluated by using Fisher exact test and the two-sample $t$ test for comparison of age at diagnosis.

Inter-observer agreement was analyzed with the Cohen $k$ statistic. The $k$ statistic was interpreted as follows: less than 0 , no agreement; $0-0.20$, slight agreement; $0.21-$ 0.40 , fair agreement; $0.41-0.60$, moderate agreement; $0.61-0.80$, substantial agreement; and 0.81-1.0, almost perfect agreement. We also estimated 95\% confidence intervals (CIs) and reported percentage agreement between the readers.

All analyses were performed by using SPSS and R (http://www.R-project.org/) software.

\section{Results}

A total of 62 patients were retrieved with 34 patients satisfying the eligibility criteria, 19 were patients excluded because another histologic type of ovarian cancer, not HGSC was found on pathology examination and 9 excluded, as they were not staged as FIGO III or IV.

The median age of the 34 women was 60 years (range, 41-80 years), 10 (29\%) of whom had BRCA1-mutant HGSC, 13 (38\%) had BRCA2-mutant HGSG, and 11 (32\%) had BRCA wild-type HGSC. For patients with $B R C A$-mutant HGSC at univariate analysis, we found a significant association between BRCA-mutant HGSC status and the age of the patients. In fact, BRCA-mutant women are younger than BRCA-wild type patients (mean age 57.0 years old vs 66.6 years old; difference in

Table 1 Demographic, clinical, and pathologic data of BRCA-mutant HGSC and BRCA-wild type patients

\begin{tabular}{lll}
\hline & BRCA wild-type $(n=11)$ & BRCA mutant $(n=23)$ \\
\hline Age, years old & & $p$ value \\
$\quad$ Mean (standard deviation) & $66.6(9.9)$ & $57.0(10.0)$ \\
Peritoneal metastasis pattern on CT or MRI, $n(\%)$ & & $14(61 \%)$ \\
$\quad$ Nodular & $7(64 \%)$ & $9(39 \%)$ \\
Infiltrative & $4(36 \%)$ & $11(65 \%)$ \\
Intraepithelial lymphocytes on pathology examination, $n(\%)^{*}$ & & $6(35 \%)$ \\
$\quad$ Present & $7(70 \%)$ & 1.000 \\
$\quad$ Absent & $3(30 \%)$ & 1.000 \\
\hline *Informa
\end{tabular}

*Information on intraepithelial lymphocytes not available in 1 BRCA wild-type carrier and in 6 BRCA mutant patients 


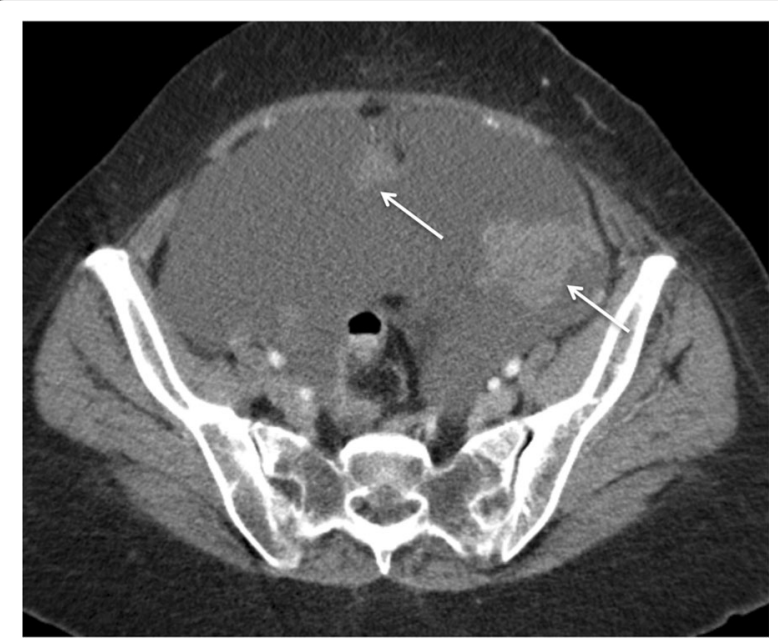

Fig. 1 Axial CT post-contrast image of the pelvis showing a nodular enhancing peritoneal metastasis pattern in a BRCA-1 mutant patient (arrows)

means 9.59 years, $95 \%$ CI: $2.15-17.04$ years; $p=0.013$ ) (Fig. 4).

The imaging results were very similar between the 3 observers with an inter-observer agreement with respect to CT/MRI features was almost perfect $(k=$ $0.82-1.00)$.

In univariate analysis, no significant associations were found between the different groups of patients (BRCA1mutant HGSC, BRCA2-mutant HGSC, and BRCA wild- type) and CT/MRI features of ovarian tumors, morphology of peritoneal metastasis $(p=0.679)$ and pathologic characteristics.

The imaging features of the peritoneal metastasis (nodular vs infiltrative) were similar in the BRCA-mutant and BCRA wild-type groups with BRCA-mutant patients having a nodular pattern of growth in $61 \%$ of the cases $(n=14)$ and BRCA wild-type in $64 \%$ of the cases $(n=7)$ (Table 1).

There was also no significant association found between the presence of intra- and extra-tumor inflammation (Fig. 3a, b) and BRCA mutant or wildtype patients at histologic analysis.

The imaging features of the primary tumors (HGSC) were similar between BRCA1-mutant, BRCA2-mutant, and sporadic cases on our series. On CT and MRI, we found heterogeneously enhancing solid masses of the ovary/fallopian tube with scattered areas of hemorrhage, cystic changes, and papillary projections (Figs. 5 and 6).

The median follow-up time in living patients was 4.2 years (range, $0.02-11.87$ years) and the median overall survival was 6.79 years in all samples, 5.85 years in BRCA-wild type patients and 7.67 in BRCA-mutant patients $(p=0.175$; Fig. 7$)$. There were 15 deaths.

\section{Discussion}

Not much is known about the imaging manifestations of certain genomic alterations in HGSC. In this study, we

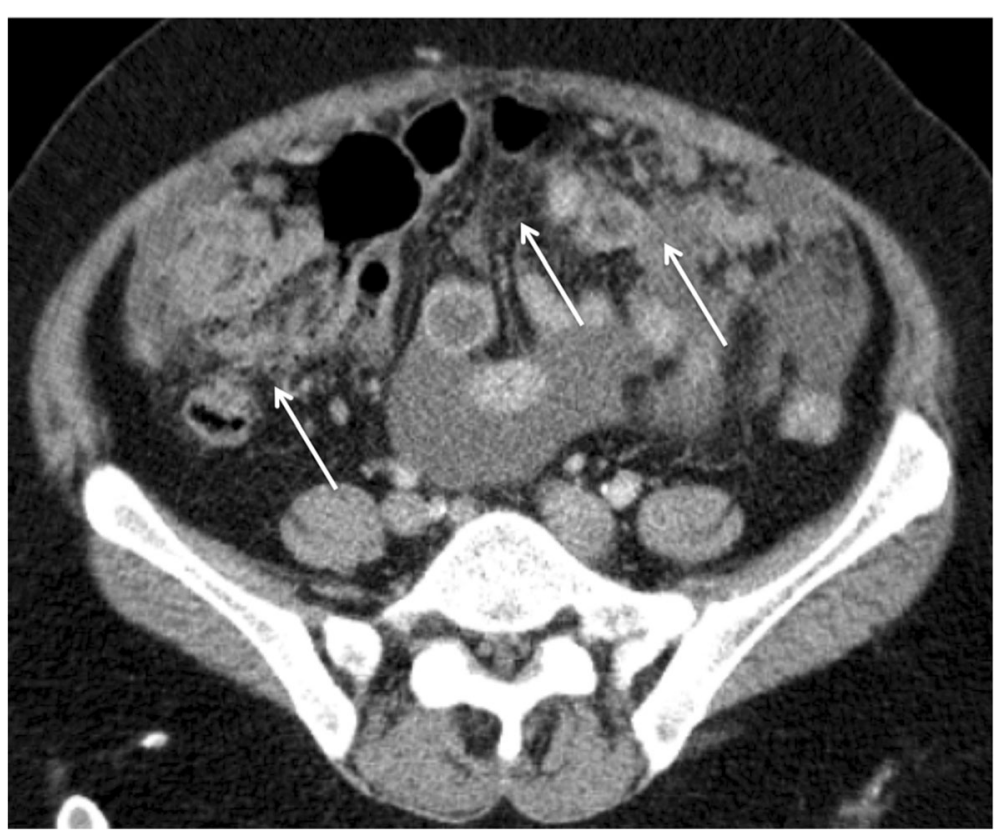

Fig. 2 Axial CT post-contrast image of the pelvis showing pelvic effusion and an infiltrative peritoneal metastasis pattern, no individualized peritoneal nodules were visualized in a BRCA-wild type patient (arrows) 


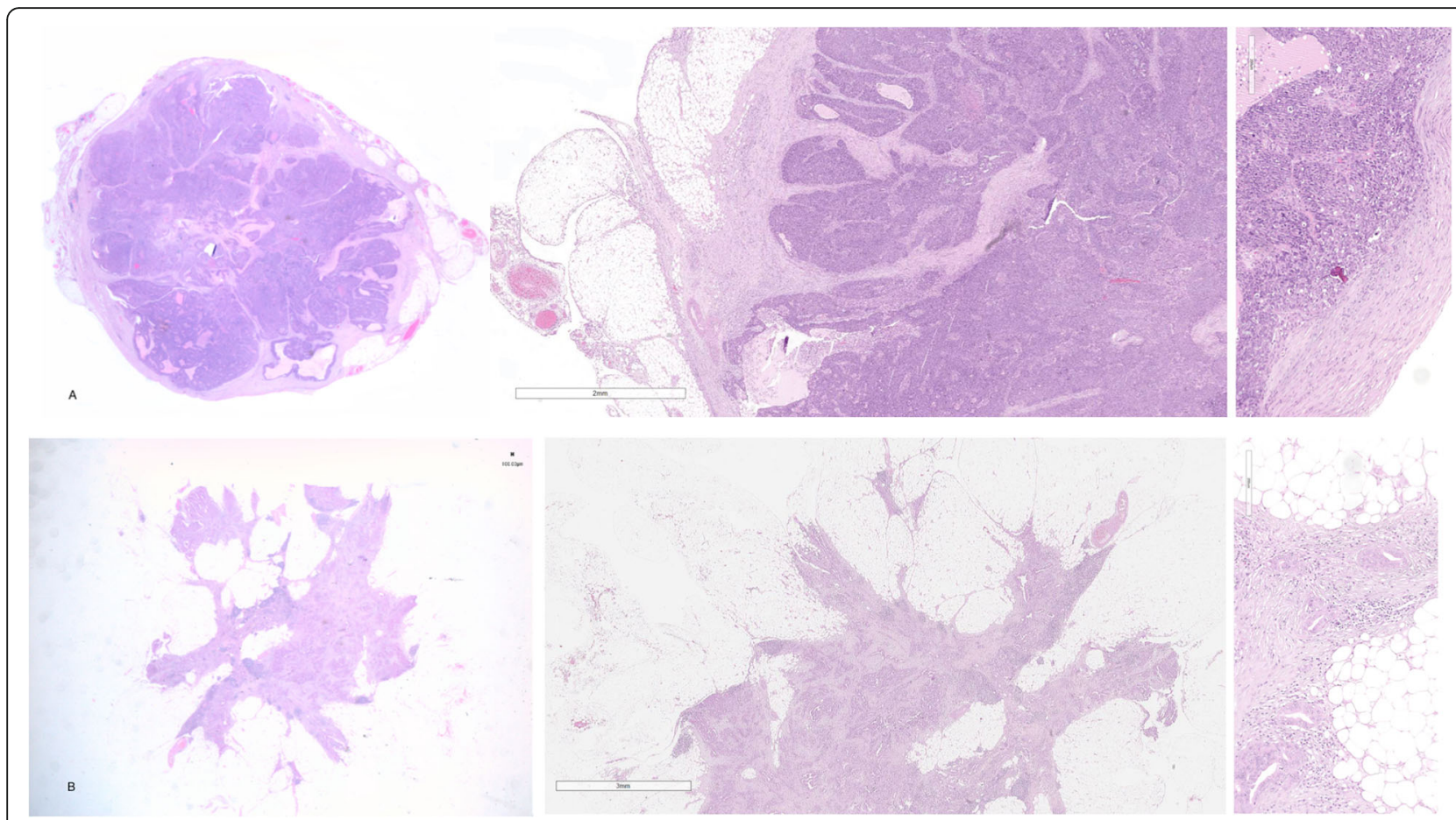

Fig. 3 a Expansile metastatic tumor growth in the omentum + inflammatory infiltrate inside the tumor-poor. b Infiltrating metastatic tumor growth in the omentum + inflammatory infiltrate inside the tumor-rich

found that CT/MRI features of HGSC do not differ with the $B R C A$ mutation status.

Imaging features as the pattern of distribution of peritoneal metastasis as well as some pathological features have already been found to be associated with gene mutation status and its correlation with primary cytoreductive surgery [19-21].

In our series, we did not identify any significant association between imaging nor histopathologic features of primary ovarian tumors, nor its omental metastases (nodular or infiltrative pattern), and gene mutation status. There were also no clear differences in the microscopic pattern of peritoneal metastases between BRCA1 or BRCA2 mutation carriers.

Regarding clinical features, $B R C A$-associated ovarian carcinomas have been reported to manifest an average of $5-10$ years earlier than sporadic ovarian carcinomas [1]. Our series confirms this tendency as we had a

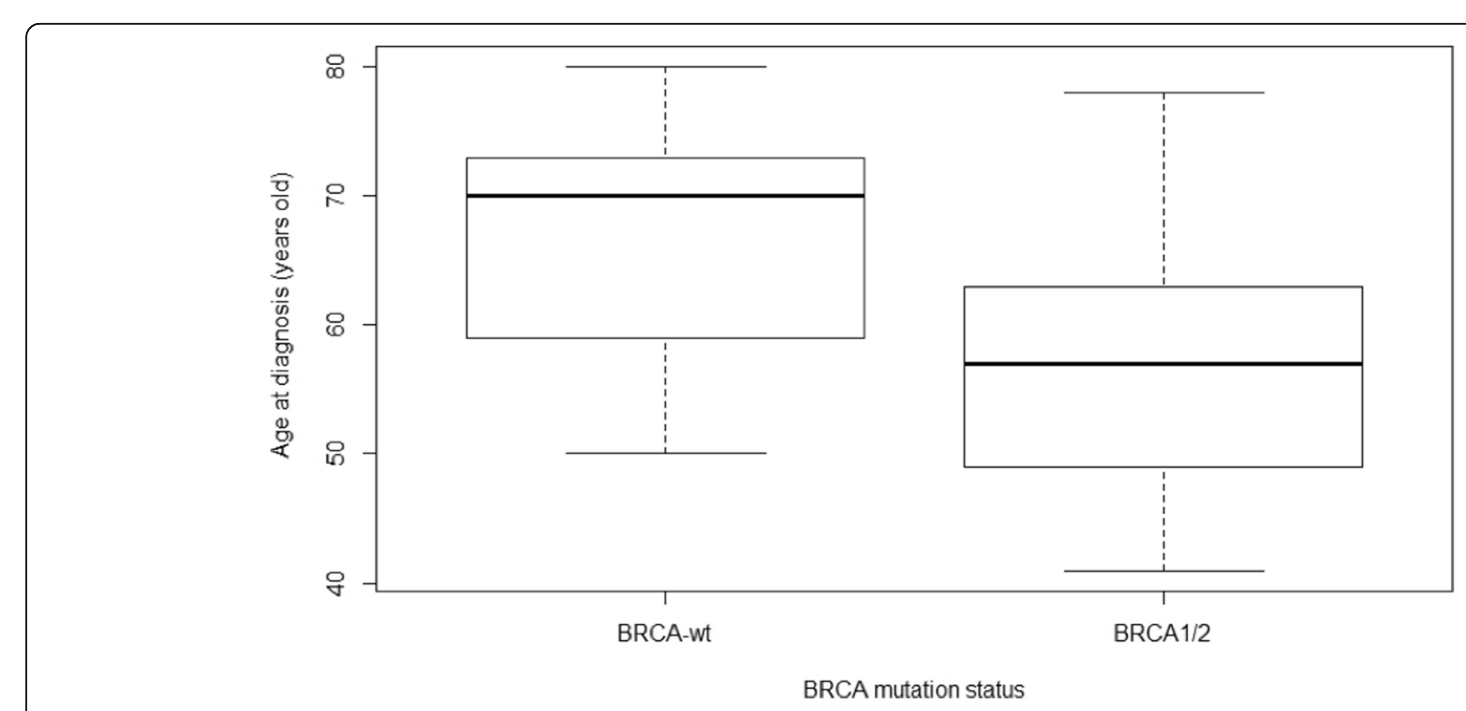

Fig. 4 Comparison between the age of the patients at diagnosis of BRCA-mutant HGSC and BRCA-wild type 


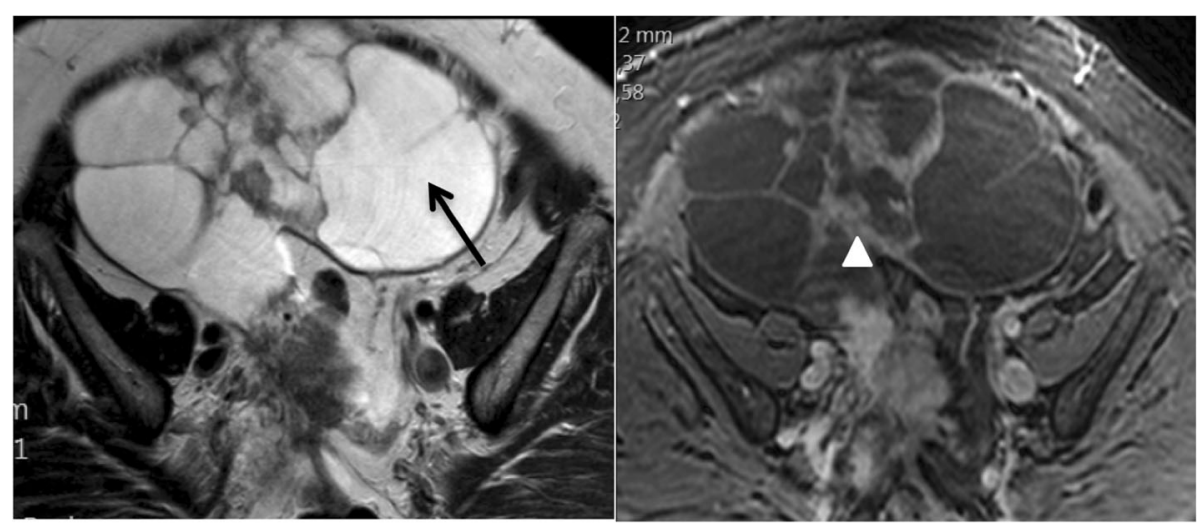

Fig. $5 \mathrm{MRI}$ T2-weighted (left) and T1-weighted fat sat post-contrast (right) images showing a heterogeneously enhancing solid mass with cystic changes (arrow) and papillary projections (arrowhead), corresponding to the primary HGSC of a BRCA mutated patient

significant association between BRCA-mutant HGSC status and the age of the patients.

Although we could not find a significant statistical difference between the overall survival of patients BRCAwild type and BRCA-mutant, we found a tendency for the latter group of patients to have a better overall survival. This is also in consonance with the literature, as it was found that $B R C A$-associated ovarian cancers tend to be more sensitive to platinum agents and poly (adenosine 5'-diphosphate-ribose) polymerase inhibitors than sporadic cancers, as well as to unique tumor biology that confers survival advantage independent of chemotherapy sensitivity [22, 23].

Our study had some limitations as it was a retrospective study with few patients with HGSC not submitted to any kind of therapy (surgery or chemotherapy) prior to the referral to our institution and tested for genetic mutations. Furthermore, the patients referred for genetic risk counseling to our institution enter on a screening program that enables us to diagnose ovarian cancer in early stages. As a result, there were patients that potentially benefited from this close follow-up program and not included in this study as they were diagnosed in an early stage. Thus, we have evaluated a small group of patients tested for BRCA1 mutation, BRCA2 mutation, and BRCA-wild type with FIGO stage III/IV, a relatively small sample size, potentially hindering the power of the study. For that reason, larger study populations are warranted to confirm our findings.

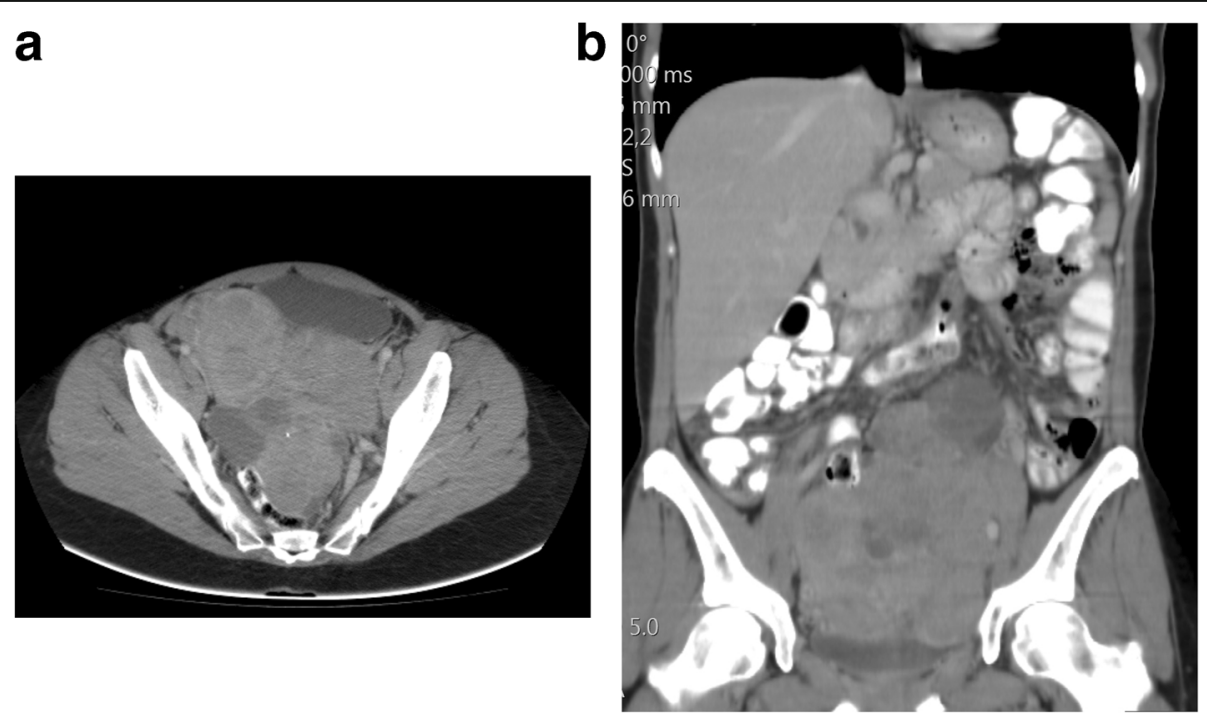

Fig. 6 Axial (left) and Coronal (right) CT post-contrast image of the abdomen and pelvis showing heterogeneously enhancing solid mass, corresponding to a primary HGSC of a BRCA 2 mutated patient 


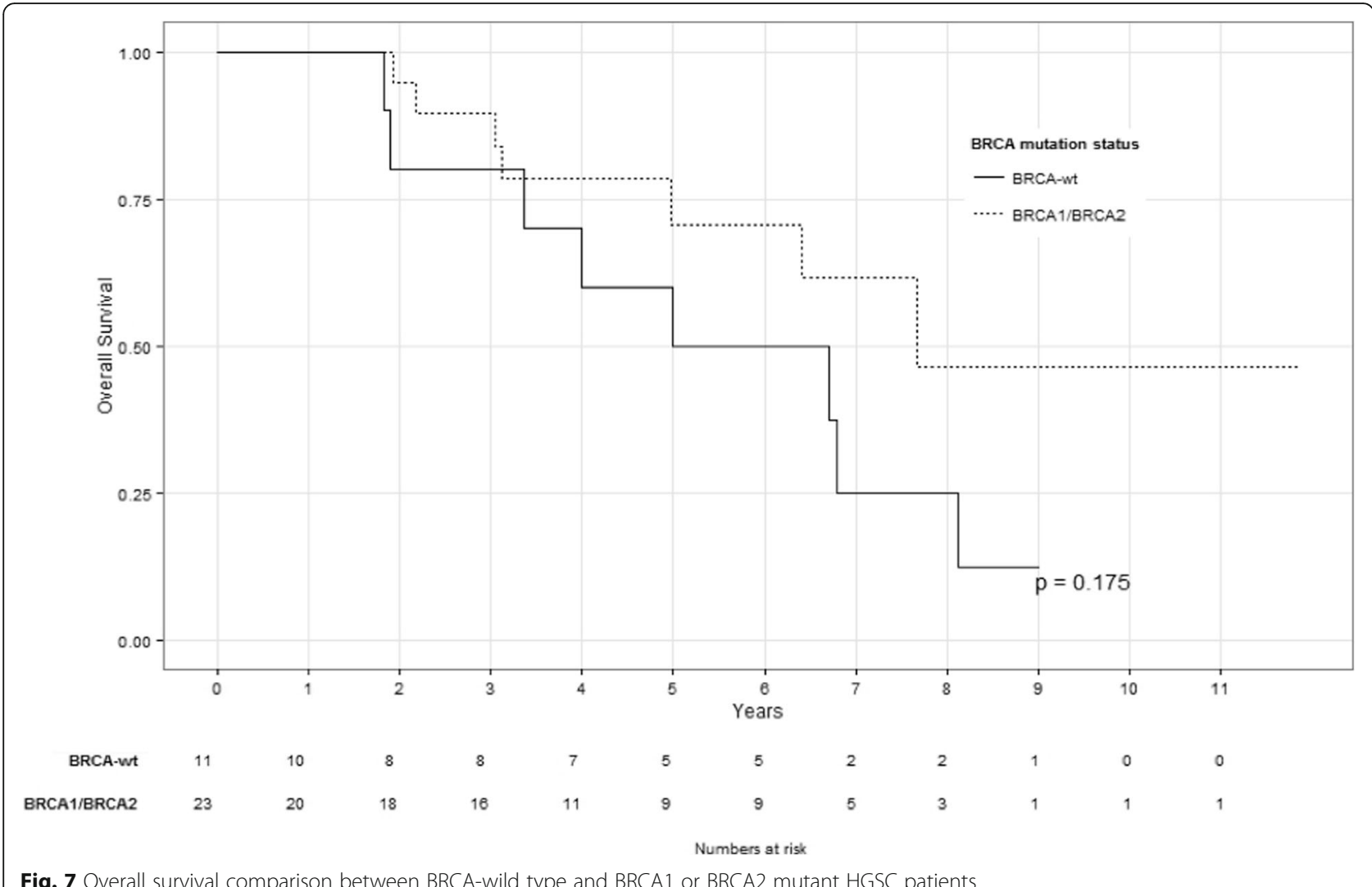

\section{Conclusion}

We could not demonstrate an association between BRCA mutation status and differences in primary tumors or peritoneal metastases pattern in CT or MRI as well as in histopathological morphology in contrast to what is stated in the most recent literature regarding the pattern of distribution of peritoneal metastasis. Consequently, the criteria described are probably the observer's dependent or not easily reproducible.

However, given the substantial prognostic and therapeutic implications of $B R C A$ mutation status it is important to continue pursuing imaging or histopathologic differences between genetic mutated and wild-type ovarian cancers that help us to provide an optimal personalized cancer treatment strategy and to develop precision medicine in the future.

\section{Abbreviations}

HGSC: High-grade serous carcinoma; Cl: Confidence interval; CT: Computed tomography; MRI: Magnetic resonance imaging

\section{Acknowledgements}

"Not applicable."

\section{Authors' contributions}

FV provided the database of patients included in this article (BRCA tested patients with HGSC). ACV, NA, and TMC performed all CT and/or MRI studies. ED and AF performed the histological examination of all surgical specimens. SE performed all statistical analyses. ACV, NA, MR, and AF were major contributors in writing the manuscript. All authors read and approved the final manuscript.

\section{Funding}

There were no external funding sources for the realization of this paper.

\section{Availability of data and materials}

The datasets analyzed during the current study are not publicly available due to the confidentiality of genetic tests performed to our subjects of study but are available from the corresponding author on reasonable request.

\section{Ethics approval and consent to participate}

Institutional review board approval was obtained and written informed consent was waived (UIC/1199). The authors declare that they have followed the protocols of their work center. Patient data confidentiality was respected.

\section{Consent for publication}

A written informed consent was waived by the institutional review board (UIC/1199). The authors declare that the procedures followed were in accordance with the regulations of the relevant clinical research ethics committee and with those of the code of ethics of the World Medical Association (Declaration of Helsinki).

\section{Competing interests}

The authors do not report any financial or personal connections with other persons or organizations, which might negatively affect the contents of this publication and/or claim authorship rights to this publication.

\section{Author details}

'Radiology Department, Instituto Português de Oncologia de Lisboa Francisco Gentil, Lisbon, Portugal. ${ }^{2}$ Radiology Department, Hospital do Espírito Santo de Évora, EPE, Évora, Portugal. ${ }^{3}$ Radiology Department, Centro Hospitalar e Universitário de Lisboa Central, EPE, Lisbon, Portugal. ${ }^{4}$ Pathology Department, Hospital Israelita Albert Einstein, São Paulo, Brazil. ${ }^{5}$ Radiology 
Department, Hospital CUF, Lisbon, Portugal. ${ }^{6}$ Department of Statistics, Instituto Português de Oncologia de Lisboa Francisco Gentil, Lisbon, Portugal. ${ }^{7}$ Oncology Department, Instituto Português de Oncologia de Lisboa Francisco Gentil, Lisbon, Portugal. ${ }^{8}$ Pathology Department, Instituto Português de Oncologia de Lisboa Francisco Gentil, Lisbon, Portugal.

Received: 18 December 2019 Accepted: 15 April 2020

Published online: 28 April 2020

\section{References}

1. Liu G, Yang D, Sun Y, Shmulevich I, Xue F, Sood AK et al (2012) Differing clinical impact of BRCA1 and BRCA2 mutations in serous ovarian cancer. Pharmacogenomics. 13(13):1523-1535

2. Siegel RL, Miller KD, Jemal A (2019) Cancer statistics, 2019. CA Cancer J Clin. 69(1):7-34

3. Lisio MA, Fu L, Goyeneche A, Gao ZH, Telleria C (2019) High-grade serous ovarian cancer: basic sciences, clinical and therapeutic standpoints. Int J Mol Sci 20(4):952. https://doi.org/10.3390/ijms20040952

4. Lee MV, Katabathina VS, Bowerson ML, Mityul MI, Shetty AS, Elsayes KM et al (2017) BRCA -associated cancers: role of imaging in screening, diagnosis, and management. RadioGraphics. 37(4):1005-1023

5. Gadducci A, Guarneri V, Peccatori FA, Ronzino G, Scandurra G, Zamagni C et al (2019) Current strategies for the targeted treatment of high-grade serous epithelial ovarian cancer and relevance of BRCA mutational status. $J$ Ovarian Res. 12(1):1-8

6. Control U (2014) for IC. Epithelial ovarian cancer. Rev Cancer Med WHO List Essent Med. 42(2010):1-9

7. Hanley GE, McAlpine JN, Miller D, Huntsman D, Schrader KA, Blake Gilks C et al (2018) A population-based analysis of germline BRCA1 and BRCA2 testing among ovarian cancer patients in an era of histotype-specific approaches to ovarian cancer prevention. BMC Cancer. 18(1):1-8

8. Girolimetti G, Perrone AM, Santini D, Barbieri E, Guerra F, Ferrari $S$ et al (2014) BRCA-associated ovarian cancer: from molecular genetics to risk management. Biomed Res Int. 2014:1-11

9. Rizzo S, Botta F, Raimondi S, Origgi D, Buscarino V, Colarieti A et al (2018) Radiomics of high-grade serous ovarian cancer: association between quantitative CT features, residual tumour and disease progression within 12 months. Eur Radiol. 28(11):4849-4859

10. Nougaret S, Lakhman Y, Gönen M, et al (2017) High-grade serous ovarian cancer: associations between. 285(2):472-481. https://doi.org/10.1148/radiol. 2017161697

11. Hynninen J, Kemppainen J, Lavonius M, Virtanen J, Matomäki J, Oksa S, et al. A prospective comparison of integrated FDG-PET/contrast-enhanced CT and contrast-enhanced CT for pretreatment imaging of advanced epithelial ovarian cancer. Gynecol Oncol [Internet]. 2013;131(2):389-394. Available from: https://doi.org/10.1016/j.ygyno.2013.08.023

12. Forstner R, Hricak H, Occhipinti K, Powell B, Frankel S, Stern J (1995) Ovarian cancer: staging with CT and MR imaging. Radiology. 5:619-626

13. Meier A, Veeraraghavan $H$, Nougaret $\mathrm{S}$, et al (2019) Association between CTtexture-derived tumor heterogeneity, outcomes, and BRCA mutation status in patients with high-grade serous ovarian cancer. Abdom Radiol (NY). 44(6):2040-2047. https://doi.org/10.1007/s00261-018-1840-5

14. Suidan RS, Ramirez PT, Sarasohn DM, Teitcher JB, Mironov S, lyer RB et al (2014) A multicenter prospective trial evaluating the ability of preoperative computed tomography scan and serum CA-125 to predict suboptimal cytoreduction at primary debulking surgery for advanced ovarian, fallopian tube, and peritoneal cancer. Gynecol Oncol. 134(3):455-461

15. Zivanovic O, Sima CS, lasonos A, Hoskins WJ, Pingle PR, Mario MM Leitao YS Jr, Abu-Rustum NR, Barakat DSC RR (2012) The effect of primary cytoreduction on outcomes of patients with FIGO stage IIIC ovarian cancer stratified by the initial tumor burden in the upper abdomen cephalad to the greater omentum. Gynecol Oncol. 17(10):1310-1314

16. Nougaret $\mathrm{S}$, Lakhman $Y$, Gönen $M$, Goldman DA, Miccò M, D'Anastasi M et al (2017) High-grade serous ovarian cancer: associations between BRCA mutation status, CT imaging phenotypes, and clinical outcomes. Radiology. 285(2):472-481

17. Gilks CB, lonescu DN, Kalloger SE, Köbel M, Irving J, Clarke B et al (2008) Tumor cell type can be reproducibly diagnosed and is of independent prognostic significance in patients with maximally debulked ovarian carcinoma. Hum Pathol. 39(8):1239-1251
18. Hussein YR, Ducie JA, Arnold AG, Kauff ND, Vargas-Alvarez HA, Sala E et al (2016) Invasion patterns of metastatic extrauterine high-grade serous carcinoma with BRCA germline mutation and correlation with clinical outcomes. Am J Surg Pathol. 40(3):404-409

19. Soslow RA, Han G, Park KJ, Garg K, Olvera N, Spriggs DR et al (2012) Morphologic patterns associated with BRCA1 and BRCA2 genotype in ovarian carcinoma. Mod Pathol. 25(4):625-636

20. Vargas HA, Miccò M, Hong Sl, et al (2015) Association between morphologic CT imaging traits and prognostically relevant gene signatures in women with high-grade serous ovarian cancer: a hypothesis-generating study. Radiology. 274(3):742-751. https://doi.org/10.1148/radiol.14141477

21. Reyes MC, Arnold AG, Kauff ND, Levine DA, Soslow RA (2014) Invasion patterns of metastatic high-grade serous carcinoma of ovary or fallopian tube associated with BRCA deficiency. Mod Pathol. 27(10):1405-1411

22. Yang D, Khan S, Sun Y, Hess K, Shmulevich I, Sood AKZW (2012) Association between BRCA2 but not BRCA1 mutations and beneficial survival, chemotherapy sensitivity, and gene mutator phenotype in patients with ovarian cancer. JAMA. 32(4):580-591

23. Gallagher DJ, Konner JA, Bell-McGuinn KM, Bhatia J, Sabbatini P, Aghajanian CA et al (2011) Survival in epithelial ovarian cancer: a multivariate analysis incorporating BRCA mutation status and platinum sensitivity. Ann Oncol. 22(5):1127-1132

\section{Publisher's Note}

Springer Nature remains neutral with regard to jurisdictional claims in published maps and institutional affiliations.

\section{Submit your manuscript to a SpringerOpen ${ }^{\circ}$ journal and benefit from:}

- Convenient online submission

- Rigorous peer review

- Open access: articles freely available online

High visibility within the field

- Retaining the copyright to your article

Submit your next manuscript at $\boldsymbol{\nabla}$ springeropen.com 